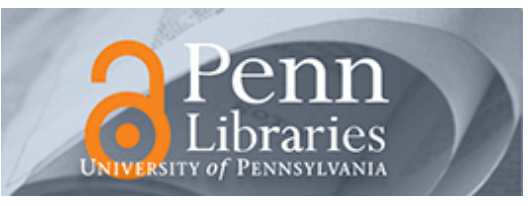

University of Pennsylvania

ScholarlyCommons

7-20-2006

\title{
Electrospun Hybrid Organic/Inorganic Semiconductor Schottky Nanodiode
}

\author{
Nicholas J. Pinto \\ University of Puerto Rico - Humacao \\ Rosana González \\ University of Puerto Rico - Humacao
}

Alan T. Johnson

University of Pennsylvania

Alan G. MacDiarmid

University of Pennsylvania

Follow this and additional works at: https://repository.upenn.edu/physics_papers

Part of the Physics Commons

\section{Recommended Citation}

Pinto, N. J., González, R., Johnson, A. T., \& MacDiarmid, A. G. (2006). Electrospun Hybrid Organic/ Inorganic Semiconductor Schottky Nanodiode. Retrieved from https://repository.upenn.edu/

physics_papers/205

Suggested Citation:

Pinto, N.J., González, R., Johnson, A.T. and MacDiarmid, A.G. (2006). Electrospun hybrid organic/inorganic semiconductor Schottky nanodiode. Applied Physics Letters 89, 033505.

(C) 2006 American Institute of Physics. This article may be downloaded for personal use only. Any other use requires prior permission of the author and the American Institute of Physics. The following article appeared in Applied Physics Letters and may be found at http://dx.doi.org/10.1063/1.2227758

This paper is posted at ScholarlyCommons. https://repository.upenn.edu/physics_papers/205

For more information, please contact repository@pobox.upenn.edu. 


\title{
Electrospun Hybrid Organic/Inorganic Semiconductor Schottky Nanodiode
}

\author{
Abstract \\ We report on a simple method to fabricate, under ambient conditions and within seconds, Schottky \\ nanodiodes using electrospun polyaniline nanofibers and an inorganic n-doped semiconductor. In \\ addition to being a rectifier, the advantage of our design is the complete exposure of the rectifying \\ nanojunction to the surrounding environment, making them attractive candidates in the potential \\ fabrication of low power, supersensitive, and rapid response sensors as well. The diode parameters were \\ calculated assuming the standard thermionic emission model of a Schottky junction, and the use of this \\ junction as a gas sensor was examined. \\ Disciplines \\ Physical Sciences and Mathematics | Physics

\section{Comments} \\ Suggested Citation: \\ Pinto, N.J., González, R., Johnson, A.T. and MacDiarmid, A.G. (2006). Electrospun hybrid organic/inorganic \\ semiconductor Schottky nanodiode. Applied Physics Letters 89, 033505. \\ (C) 2006 American Institute of Physics. This article may be downloaded for personal use only. Any other \\ use requires prior permission of the author and the American Institute of Physics. The following article \\ appeared in Applied Physics Letters and may be found at http://dx.doi.org/10.1063/1.2227758
}




\title{
Electrospun hybrid organic/inorganic semiconductor Schottky nanodiode
}

\author{
Nicholas J. Pinto ${ }^{a)}$ and Rosana González \\ Department of Physics and Electronics, University of Puerto Rico, Humacao, Puerto Rico 00791
}

Alan T. Johnson, Jr.

Department of Physics and Astronomy, University of Pennsylvania, Philadelphia, Pennsylvania 19104

Alan G. MacDiarmid ${ }^{\mathrm{b})}$

Department of Chemistry, University of Pennsylvania, Philadelphia, Pennsylvania 19104

(Received 21 March 2006; accepted 15 June 2006; published online 20 July 2006)

\begin{abstract}
We report on a simple method to fabricate, under ambient conditions and within seconds, Schottky nanodiodes using electrospun polyaniline nanofibers and an inorganic $n$-doped semiconductor. In addition to being a rectifier, the advantage of our design is the complete exposure of the rectifying nanojunction to the surrounding environment, making them attractive candidates in the potential fabrication of low power, supersensitive, and rapid response sensors as well. The diode parameters were calculated assuming the standard thermionic emission model of a Schottky junction, and the use of this junction as a gas sensor was examined. () 2006 American Institute of Physics.
\end{abstract}

[DOI: $10.1063 / 1.2227758$ ]

Devices and sensors based on the use of $\pi$-conjugated conducting polymers are considered by many in the field to shape the next generation of cheap and disposable electronic inventions. The simplest and easiest polymer based device to fabricate is a hybrid organic/inorganic Schottky diode in which a junction of a $p$-doped polymer with an $n$-doped inorganic semiconductor is formed. This construction has been achieved via electrochemical polymerization ${ }^{1-4}$ or spin coating $^{5}$ of the polymer onto the $n$-doped semiconducting substrate. Such a "wet" process, however, could result in undesirable chemical reactions with the substrate, leading to partial degradation of the semiconductor at the interface with the polymer causing detrimental effects on device operation. Furthermore, a large fraction of the resulting polymer/ inorganic active semiconductor junction, which is typically two dimensional (2D), is unexposed, being sandwiched between layers in the final device layout and thus not optimal for use as a sensor. In such relatively large 2D structures the effective area of the junction is also uncertain. We report on a simple method to fabricate in air, and within seconds, Schottky nanodiodes using polyaniline and an inorganic $n$-doped semiconductor. The advantage of our design is the complete exposure of the rectifying nanojunction to the surrounding environment, making them attractive candidates in the potential fabrication of low power, supersensitive, and rapid response sensors and rectifiers.

The Schottky diode is prepared by using an $n$-doped $\mathrm{Si}$ wafer $(\langle 111\rangle, 0.1-1.0 \Omega \mathrm{cm})$ with a $200 \mathrm{~nm}$ thermally grown oxide layer and polyaniline (PANI). After prepatterning gold electrodes over the oxide via standard lithography and lift-off techniques the substrate is cleaved through the electrodes. The exposed cleaved surface has the edge of the gold electrode separated from the doped $\mathrm{Si}$ by the insulating oxide layer. For the polymer, $100 \mathrm{mg}$ of emeraldine base PANI was doped with $129 \mathrm{mg}$ of camphorsulfonic acid (HCSA) and dissolved in $10 \mathrm{ml} \mathrm{CHCl}_{3}$ for a period of $4 \mathrm{~h}$. The resulting

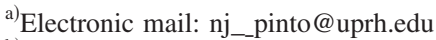

${ }^{b)}$ Also at: Departments of Chemistry and Physics, University of Texas at Dallas, Richardson, TX 75083.
}

deep green solution was filtered, and $10 \mathrm{mg}$ of polyethylene oxide (PEO) having molecular weight of 900000 was added to the solution and stirred for an additional of $2 \mathrm{~h}$. PEO was added to assist in fiber formation, and the solution was then filtered using a $0.45 \mu \mathrm{m}$ polytetra fluoroethylene (PTFE) syringe filter. Using an electrospinning technique reported earlier, ${ }^{6,7}$ individual, charged, dry, and flexible PANI nanofibers were deposited over the wafer edge, making contacts to the gold and the doped Si. These are stable with no apparent degradation or oxidation. The resulting Schottky diode is formed along the vertical edge of the substrate at the nanofiber-doped Si interface. Such a vertical orientation may offer higher levels of integration in circuitry than that provided by in-plane horizontal structures. External electrical contacts were then made, and the device current-voltage $(I-V)$ characteristics were measured via a Keithley 6517A electrometer in a vacuum of $\sim 2 \times 10^{-2}$ Torr. Figure 1 shows a schematic of the device and the external electrical circuit.

Figure 2(a) shows a top view of the cleaved substrate with the electrospun polymer nanofiber using a scanning electron microscope (SEM). The nanofiber was seen to firmly adhere to the substrate and to reach toward and over the edge. In order to measure the diameter, an atomic force microscope (AFM) was used in tapping mode to scan a portion of the fiber, as indicated in the boxed section of Fig. 2(a). The result of this measurement is shown in Figs. 2(b) and 2(c). The average fiber diameter was $\sim 70 \mathrm{~nm}$ and assumed to be that of the fiber at the Schottky nanojunction. Figure 3 shows the $I-V$ characteristic curve at $300 \mathrm{~K}$ of the

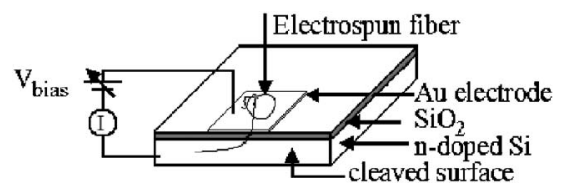

FIG. 1. Schematic of the prepatterned and then cleaved $n$-doped $\mathrm{Si} / \mathrm{SiO}_{2}$ substrate and the electrospun polymer nanofiber making contacts to the gold electrode above and to the doped Si below the oxide layer. The real fiber is flexible and does not fracture as it bends over the substrate edge. The external electrical connections are also shown. 


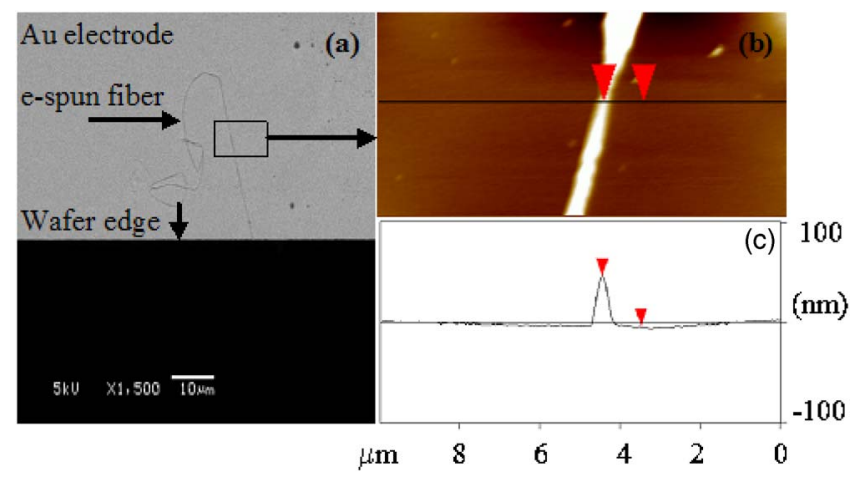

FIG. 2. (Color online) (a) Scanning electron microscope image showing a top view of the electrospun nanofiber lying on the gold electrode and extending to and over the wafer edge. (b) Atomic force microscope height image of a section of the fiber indicated by the box in (a) together with a height profile along a selected dark line and shown in part (c). The average fiber diameter was $\sim 70 \mathrm{~nm}$.

device shown in Fig. 2. Depending on whether the positive terminal of $V_{\text {bias }}$ was connected to the gold or to the doped $\mathrm{Si}$, the forward biased diode response lies either in the first or the third quadrant, respectively, thereby confirming the formation of Schottky barriers at the polymer $/ n$-doped semiconductor junction. Several diodes were tested, and in general the characteristic $I-V$ curves were asymmetrical with a turn on voltage in the range of $0.4-0.6 \mathrm{~V}$ and a much reduced reverse bias current that did not tend to saturate. These devices exhibited a rectifying behavior, and the ratio of the forward to reverse current at a bias voltage of $\pm 1 \mathrm{~V}$ for this device was calculated to be 160 which was limited in part due to the low fiber conductivity and the series resistance of the semiconductor. Pretreating the substrate with dilute HF prior to fiber deposition is expected to improve the device rectification ratio. ${ }^{4}$ In order to verify the type of contact between the polymer and the gold electrode, another polymer nanofiber $(\sim 150 \mathrm{~nm}$ diameter) was electrospun so as to con-

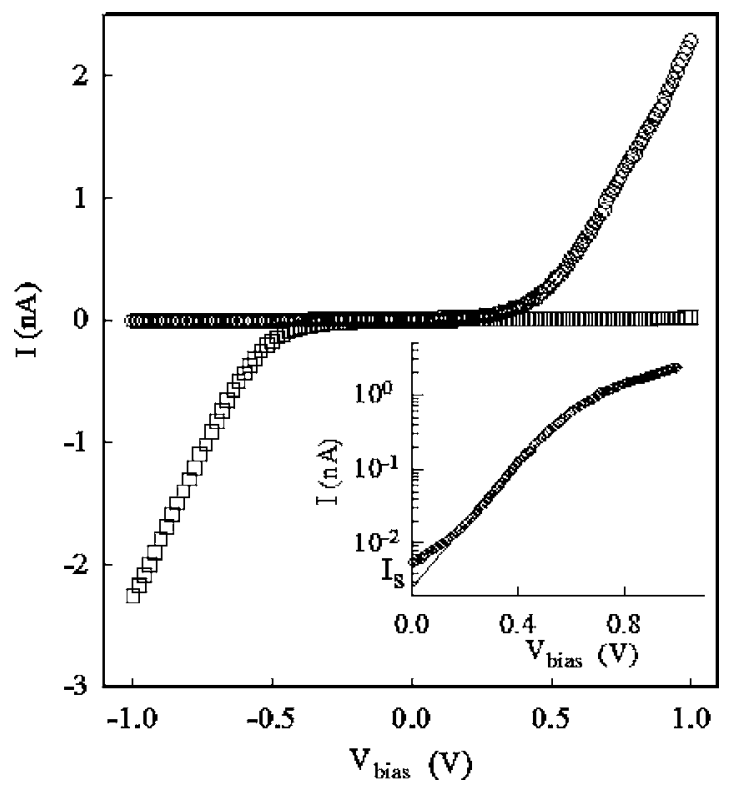

FIG. 3. Current-voltage characteristics at $300 \mathrm{~K}$ of the Schottky diode shown in the previous figure when the positive terminal of $V_{\text {bias }}$ was connected to the gold electrode $(O)$ and when the positive terminal of $V_{\text {bias }}$ was connected to the doped $\mathrm{Si}(\square)$. Inset: Semilog plot of the forward bias current as a function of forward bias voltage for the case when the positive

terminal of $V_{\text {bias }}$ was connected to the gold electrode.
Downloaded 23 Jun 2011 to 130.91.117.41. Redistribution subject to AIP license or copyright; see http://apl.aip.org/about/rights_and_permissions

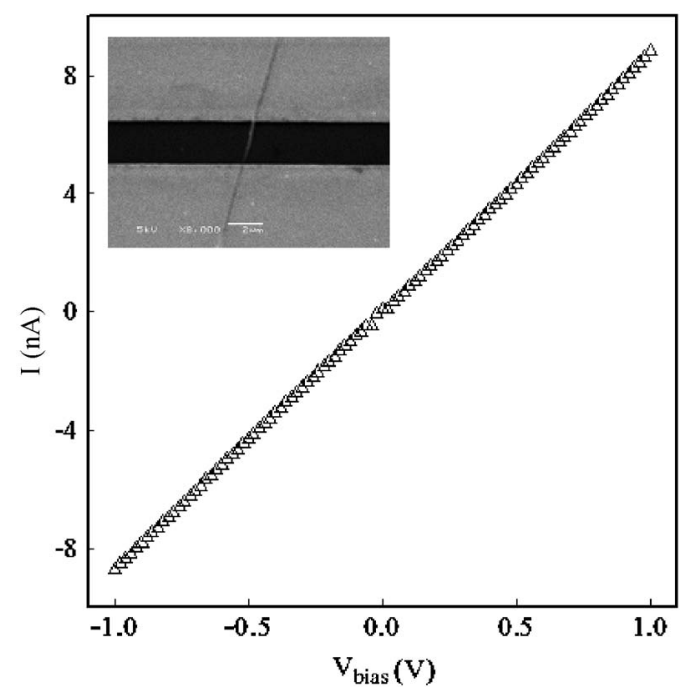

FIG. 4. I-V characteristic curve at $300 \mathrm{~K}$ for a single $150 \mathrm{~nm}$ diameter electrospun polyaniline fiber that makes contacts to two gold electrodes having a $2 \mu \mathrm{m}$ separation. The linear response indicates Ohmic contacts, and we calculate a fiber conductivity of $\sim 5 \times 10^{-2} \mathrm{~S} / \mathrm{cm}$. Inset: SEM image of the fiber bridging the two electrodes as seen from above.

tact two gold electrodes on the same substrate as that of the previous diode. Figure 4 shows the corresponding $I$ - $V$ curve for this fiber together with a SEM image in the insert to Fig. 4. A linear response confirms that the polymer contact with the gold electrode is indeed Ohmic, implying a work function close to that of gold $(5.1 \mathrm{eV})$, and the rectifying behavior seen in Fig. 3 arises primarily from the polymer-doped $\mathrm{Si}$ interface. An analysis of the fiber resistance obtained from Fig. 4 combined with the fiber dimensions yielded a conductivity of $\sim 5 \times 10^{-2} \mathrm{~S} / \mathrm{cm}$, consistent with earlier reports. ${ }^{6,7}$

In order to quantitatively analyze the diode characteristics, we assume the standard thermionic emission model of a Schottky junction. In this model the forward biased current should obey the well known relationship ${ }^{8-10}$

$$
J=J_{S} e^{-q V / n k T},
$$

where

$$
J_{s}=A^{*} T^{2} e^{q \varphi_{b} / k T},
$$

with

$$
A^{*}=\frac{4 \pi q m^{*} k^{2}}{h^{3}},
$$

where $J$ is the current density (current/area), $J_{s}$ is the saturation current density, $q$ is the absolute electron charge, $m^{*}$ is the effective electron mass, $h$ is Planck's constant, $k$ is Boltzmann's constant, $T$ is the absolute temperature, $\varphi_{b}$ is the barrier height, and $n$ is the ideality factor which takes into account corrections to the original simple model, e.g., imageforce barrier lowering. Richardson's constant $\left(A^{*}\right)$ is calculated to be $120 \mathrm{~A}^{2} / \mathrm{K}^{2} \mathrm{~cm}^{2}$. The inset to Fig. 3 shows the semilogarithmic plot of the diode current versus applied voltage under forward bias conditions. At low biases a linear variation of the current is indeed observed, consistent with Eq. (1), while the deviation from linearity at higher bias voltages is generally related to Ohmic losses in the bulk of the semiconductor and possibly to contact resistance. Extrapolating the linear portion of the semilog plot to zero bias yields a saturation current density of $6.5 \times 10^{-2} \mathrm{~A} / \mathrm{cm}^{2}$, and icense or copyright; see http://apl.aip.org/about/rights_and_permissions 


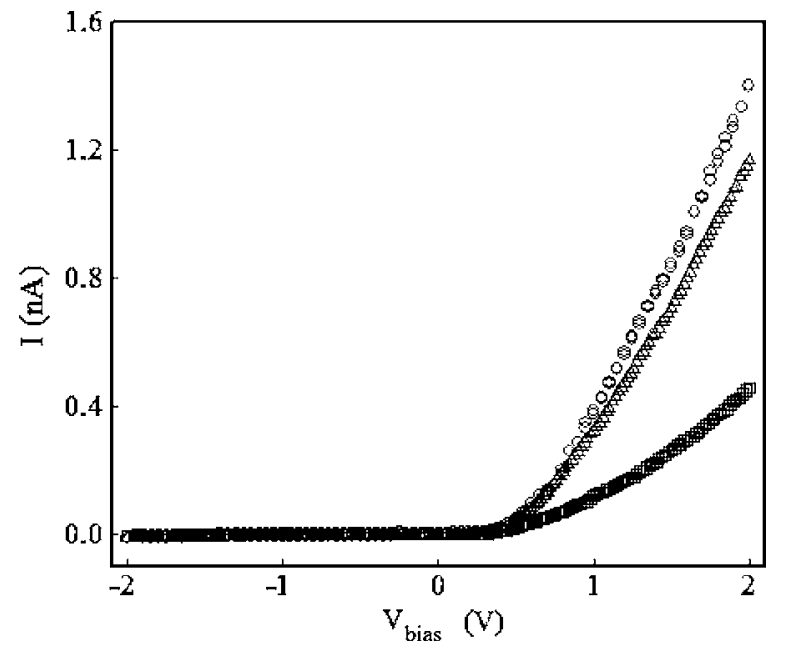

FIG. 5. $I-V$ characteristic curves for a Schottky nanodiode when used as a gas sensor and measured in the following sequence: $(O)$ as prepared sample measured in vacuum, $(\square)$ a few seconds after exposure to ammonia vapor, and $(\triangle)$ after $5 \mathrm{~h}$ of pumping.

the diode ideality factor calculated from the slope of the linear portion of the plot is $n \approx 4[n=(q / k T)(\partial V / \partial \ln J)]$. Using Eq. (2) we calculate the barrier height of $0.49 \mathrm{eV}$. While the rectification ratio is low and the ideality factor is high compared to all inorganic Schottky diodes, the present design, size, and simple fabrication technique nevertheless make them attractive candidates for use as sensors as well.

It is well known that the conductivity of polyaniline is affected by the dedoping effects of a base such as ammonium hydroxide $^{11,12}\left(\mathrm{NH}_{4} \mathrm{OH}\right)$. This motivated us to test the diode response when exposed to ammonia gas. Figure 5 shows the results of this experiment in an as prepared device measured in vacuum, after ammonia exposure ${ }^{13}$ and after pumping out the ammonia. Due to the large surface to volume ratio of the fiber, the change in the diode response was instantaneous upon exposure to ammonia with nearly complete recovery of the current upon pumping, thereby making it a reusable sensor. The recovery response can be improved by using a more efficient vacuum system. As seen from Fig. 5, the diode turn on voltage is not affected by the sensing action, possibly due to the presence of surface states at the junction that control device operation. The freshly cleaved Si substrate is susceptible to containing several dangling $\mathrm{Si}$ bonds that would interact with the fiber upon contact, thereby pinning the Fermi levels. Such pinning would make the application of this device as a sensor more reliable due to a fixed turn on voltage even in the presence of harmful gases.

In summary, we present a facile method of fabricating Schottky nanodiodes using the electrospinning technique and analyze the diode characteristics using the standard thermionic emission model of a Schottky junction. The high surface to volume ratio of the nanofiber makes these devices attractive candidates in the potential fabrication of low power, supersensitive, and rapid response reusable sensors. Attempts to measure the true junction capacitance were unreliable, in part due to the extremely small diode active junction area. An advantage of having a small junction capacitance, however, is the use of such diodes in high frequency rectifiers. Additional work to electrically characterize these devices as a function of fiber diameter and temperature is currently underway.

This work was supported in part by NSF under Grant Nos. 0402766 and 0353730 and by the Petroleum Research Fund under Grant No. 38880-B7. One of the authors (N.J.P.) is grateful to NASA-GRC for providing the prepatterned substrates.

${ }^{1}$ O. Inganäs, T. Skotheim, and I. Lundström, J. Appl. Phys. 54, 3636 (1983).

${ }^{2}$ M. J. Sailor, F. L. Klavetter, R. H. Grubbs, and N. S. Lewis, Nature (London) 346, 155 (1990).

${ }^{3}$ M. C. Lonergan, Science 278, 2103 (1997).

${ }^{4}$ Le. T. T. Tuyen, K. P. Kamloth, and H.-D. Liess, Thin Solid Films 292, 293 (1997).

${ }^{5}$ D. P. Halliday, J. W. Gray, P. N. Adams, and A. P. Monkman, Synth. Met. 102, 877 (1999).

${ }^{6}$ Y. X. Zhou, M. Freitag, J. Hone, C. Staii, A. T. Johnson, N. J. Pinto, and A. G. MacDiarmid, Appl. Phys. Lett. 83, 3800 (2003).

${ }^{7}$ N. J. Pinto, A. T. Johnson, A. G. MacDiarmid, C. H. Mueller, N. Theofylaktos, D. C. Robinson, and F. A. Miranda, Appl. Phys. Lett. 83, 4244 (2003).

${ }^{8}$ P. M. Grant, T. Tani, W. D. Gill, M. Krounbi, and T. C. Clarke, J. Appl. Phys. 52, 869 (1981).

${ }^{9}$ G. Horowitz, Adv. Mater. (Weinheim, Ger.) 2, 287 (1990).

${ }^{10}$ S. M. Sze, Physics of Semiconductor Devices (Wiley, New York, 1981), Chap. 5, p. 256.

${ }^{11}$ G. E. Asturias, A. G. MacDiarmid, R. P. McCall, and A. J. Epstein, Synth. Met. 29, E157 (1989).

${ }^{12}$ I. D. Norris, M. M. Shaker, F. K. Ko, and A. G. MacDiarmid, Synth. Met. 114, 109 (2000).

${ }^{13}$ Concentrated $\mathrm{NH}_{4} \mathrm{OH}$ was placed in a volumetric flask, and the saturated ammonia vapor formed above the solution was slowly leaked into the vacuum chamber. 